\title{
Measurement of nanotube content in pyrolytically generated carbon soot
}

\author{
Jonathan N. Coleman,*a Diarmuid F. O’Brien, ${ }^{a}$ Alan B. Dalton, ${ }^{b}$ Brendan McCarthy, ${ }^{a}$ Bernd Lahr, ${ }^{a}$ Anna \\ Drury, ${ }^{a}$ Robert C. Barklie ${ }^{a}$ and Werner J. Blau ${ }^{a}$ \\ a Materials Ireland Polymer Research Center, Department of Physics, University of Dublin, Trinity College, Dublin \\ 2, Republic of Ireland. E-mail: colemaj@tcd.ie \\ ${ }^{b}$ School of Physics, Dublin Institute of Technology, Kevin St, Dublin 8, Republic of Ireland
}

\section{Received (in Cambridge, UK) 31st July 2000, Accepted 5th September 2000 First published as an Advance Article on the web 27th September 2000}

Carbon nanotubes can be efficiently separated from impurity material in carbon soot using a conjugated polymer filtration system as monitored by EPR, allowing the calculation of purity of the crude carbon soot.

Carbon nanotubes have generated interest in all areas of science owing to their novel structural, mechanical and electronic properties. In the physical sciences nanodevices have already been demonstrated including transistors ${ }^{1}$ and rectifying heterojunctions. ${ }^{2}$ In microbiology they have been used as probes to study the structure of biomolecules ${ }^{3,4}$ and as templates for the self assembly of proteins. ${ }^{5}$ However, at present, as-produced carbon soot remains low in nanotube content. ${ }^{6}$ Furthermore, neither quantitative techniques to analyse soot content nor methods to measure nanotube content exist. This work presents the first measurement of nanotube content in impure carbon soot. Using a conjugated polymer as a nanotube 'filter', carbon nanotubes are separated from all other soot components. An absolute value for the nanotube content can then be calculated for the first time using electron paramagnetic resonance and thermogravimetric measurements. This is a vital step towards making nanotubes a practical material for novel scientific developments.

The necessity for a technique to measure nanotube content in carbon soot is apparent when the present fabrication and purification methods are examined. During nanotube production unwanted carbon species such as turbostratic graphite (TSG) and carbon onions are invariably formed. Purification by oxidation ${ }^{7}$ destroys many nanotubes and alters the electronic properties of the remaining tubes. Chromatographic techniques 8 have succeeded in purifying carbon soot but no quantitative measure of purity has been obtained. Furthermore, scale-up of these processes is problematic. The process outlined in this work approaches these issues in a novel manner via the production of a polymer nanotube composite. Thus a quantifiable purification method for carbon soot is presented which leads logically to a measurement of the purity of that soot.

In order to produce the polymer nanotube composites used in this work $80 \mathrm{mg}$ of poly $(m$-phenylene-co-2,5-dioctyloxy- $p$ phenylenevinylene) (PmPV) were mixed with $25.5 \mathrm{mg}$ of multiwall nanotube (MWNT) containing arc-generated carbon soot in $4 \mathrm{ml}$ of toluene. The PmPV was synthesised using a standard polycondensation reaction, ${ }^{9}$ while the carbon soot was generated in a Krätschmer generator. ${ }^{10}$ The mixture was sonicated for 2 min using a high power sonic tip and then for 2 $\mathrm{h}$ in a low power sonic bath to ensure complete dispersion of the Krätschmer generated carbon soot. This was carried out for seven composite solutions with identical constituents. These solutions were then allowed to stand undisturbed for various amounts of time, from $30 \mathrm{~min}$ to $90 \mathrm{~h}$. At the end of its settling time each solution was carefully decanted into a new sample bottle, leaving a black sediment at the bottom of the old bottle. These sediments were then dried and weighed.

To determine the natures of the sediment and remaining solute, EPR spectroscopy was used. This technique measures microwave-induced transitions between electron spin energy levels in the presence of a magnetic field. Unpaired electrons in different environments may be distinguished using this technique by differences in their resonance spectra. To prepare samples for EPR, $c a .0 .3 \mathrm{ml}$ of each of the separated solutions was drop cast onto spin free quartz plates, giving $\mathrm{ca} .7 \mathrm{mg}$ of solute after the solvent had evaporated. In addition all the recovered sediments were carefully weighed and $c a .7 \mathrm{mg}$ of each placed in spin free quartz tubes. EPR spectra were recorded for all samples and carbon soot. The carbon soot was dispersed in toluene in a spin free glass tube to reduce the interaction of the spins within neighbouring particles, and to match more closely the environment of the spins in a polymer host.

Fig. 1 shows EPR derivative spectra for the dispersed carbon soot, the sediment and solute samples for both the shortest and longest settling times. In all cases these spectra could well be fitted to the superposition of two symmetric absorption lines of Lorentzian shape. In the case of the dispersed carbon soot and the various sediments, $g$ values determined from the line positions, of $c a$. 2.011 and 2.020 and peak-to-peak line widths, $\Delta B_{\mathrm{pp}}$, of close to 11 and $12 \mathrm{G}$, respectively, were observed. Similar results were obtained for the solute spectra which could be fitted to two lines with $g$ values of $c a$. 2.011 and 2.020 and widths of 7 and $18 \mathrm{G}$, respectively. This demonstrates that the carbon soot consists of the same two components as are in the solutes and sediments. The variation in linewidth between sediment and solute is probably due to small environmental variations between the two phases. Two such components have been observed by other authors who attribute them to paramagnetic centers in nanotubes 6,11 and TSG.

In addition to $g$ values and linewidths, signal intensities can be measured for both the MWNT and TSG. In each case the signal intensity of the MWNT or TSG line is proportional to the mass of the MWNT or TSG present in the measured sample. Assuming that the measured fraction is representative of the whole mass of sediment or solute, the signal intensities can be normalized to represent all the unpaired spins in the total mass of sediment or solute. This normalized signal intensity $(N S I)$ is achieved by multiplying the measured signal intensity by a

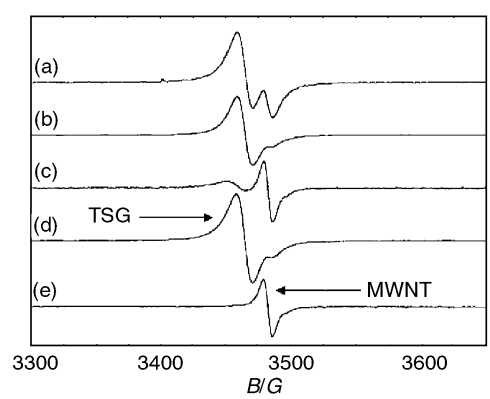

Fig. 1 EPR derivative spectra for some of the samples studied in this work EPR spectra of (a) carbon soot dispersed in toluene, (b) the sediment formed after $30 \mathrm{~min}$ settling time, (c) the solute remaining after $30 \mathrm{~min}$ settling time, (d) the sediment formed after $90 \mathrm{~h}$ settling time and (e) the solute remaining after $90 \mathrm{~h}$ settling time. Note that in all spectra except $\mathrm{E}$ two components, TSG and MWNT (denoted by arrows), are clearly present. For spectrum E the sole component present is that of the MWNT. 


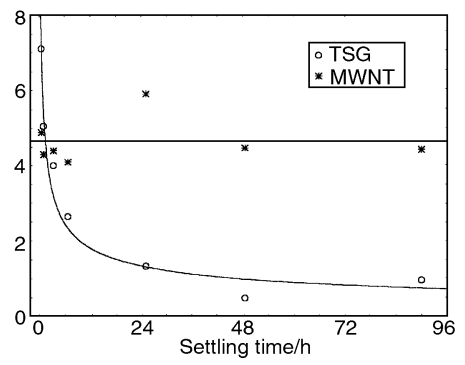

Fig. 2 Normalised signal intensities (NSI) for the nanotube and TSG components of the solute formed for various settling times. The NSI values are proportional to the entire mass of each component for each solute. Note that while the nanotube component remains approximately constant the turbostratic graphite component decreases as the TSG falls out of solution.

factor of $M_{\mathrm{T}} / M_{\mathrm{EPR}}$ where $M_{\mathrm{T}}$ is the total sediment or solute mass and $M_{\mathrm{EPR}}$ is the mass of the sample measured.

These normalized signal intensities, for both nanotube and TSG components of the solute are shown in Fig. 2 as a function of sample settling time. It is clear from this diagram that the nanotube component of the various solutes is approximately constant for all the settling times. The TSG component however shows a sharp decrease. After $48 \mathrm{~h}$ settling time there is virtually no TSG present in the solute. Therefore the PmPV holds carbon nanotubes in solution while the TSG gradually settles out to give solutions rich in nanotubes. Thus nanotubes can be effectively separated from other unwanted forms of carbon present in carbon soot.

For the $48 \mathrm{~h}$ settling time we can calculate the percentages of both MWNT and TSG that have remained in solution. This can be calculated for a given species from

$$
\%=100 \times \frac{N S I_{\text {solution }}}{N S I_{\text {solution }}+N S I_{\text {sediment }}}
$$

where $\%$ is the percentage of the given species (MWNT or TSG) in solution, $N S I_{\text {solution }}$ and $N S I_{\text {sediment }}$ are the normalized signal intensities for the same species in solution and sediment, respectively. For the $48 \mathrm{~h}$ settling sample $N S I_{\text {solution }}(\mathrm{MWNT})=$ 4.5 while $N S I_{\text {sediment }}(\mathrm{MWNT})=2.6$ (in arbitrary units). By comparison for the same sample $N S I_{\text {solution }}(\mathrm{TSG})=0.5$ while $N S I_{\text {sediment }}(\mathrm{TSG})=26.5$. Using this we can calculate that $63 \%$ of the added nanotubes go into solution while only $1.9 \%$ of the added TSG remains in solution.

While this allows us to make relative comparisons of amounts of nanotubes present in a given sample using the NSI values, EPR alone does not give us enough information to make absolute measurements of nanotube content. In order to do this we need to be able to calculate a nanotube signal intensity per unit mass, $k$, such that we can write an equation of the form

$$
S_{i}=k_{i} m_{i}
$$

where $S_{i}$ is a signal intensity for a given mass $m_{i}$ of a given species (MWNT or TSG) $i$. This will allow us to calculate the mass of nanotubes in a given sample from the EPR spectrum provided we know $k$.

In order to calculate the mass of nanotubes present in a purified composite sample with only trace amounts of TSG we use thermogravimetric analysis (TGA). In this technique the sample is heated in air and the sample mass monitored as it is oxidised. This was carried out for the carbon soot, PmPV and a highly separated solute sample (settling time $48 \mathrm{~h}$ ). The TGA traces for these samples are shown in Fig. 3. By $650{ }^{\circ} \mathrm{C}$ the PmPV sample is almost completely oxidised (except for some impurities), while the carbon soot only begins to burn at $c a$. $700{ }^{\circ} \mathrm{C}$. For the composite sample, between these two temperatures, no oxidisation occurs as demonstrated from the horizontal part of the trace in this region. Thus the stable mass in this temperature region represents nanotubes and some polymer impurities. It is possible to account for the polymer impurities and hence calculate the mass of nanotubes present in the composite using these data. For the composite solute

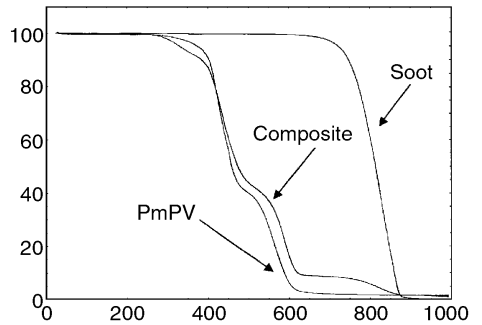

Fig. 3 Thermogravitational analysis trace for PmPV, a highly separated composite film and carbon soot. Note that PmPV stops burning at $c a .600{ }^{\circ} \mathrm{C}$ while the carbon soot only begins to burn at $750{ }^{\circ} \mathrm{C}$. For the composite sample there is no oxidation between these temperatures. This allows us to calculate the mass of nanotubes in this sample.

measured here the nanotube mass was calculated at $5.1 \%$ of the total composite mass. This allows us to calculate the actual mass of nanotubes present in the (same) sample measured by EPR. As we know the nanotube EPR signal intensity $\left(S_{n}\right)$ for this sample we can calculate a signal intensity per mass of nanotubes, $k_{n}$. This works out to be 1.04 per $\mathrm{mg}$ in our system of units.

From this it is possible to calculate the nanotube content in the carbon soot. As described above we can obtain a NSI for the nanotube component in the carbon soot. This was measured as 0.53 for the $1.5 \mathrm{mg}$ of carbon soot dispersed in toluene, giving a nanotube component in this sample of carbon soot a mass of $m_{n}=S_{n} / k_{n}=0.53 / 1.04 \mathrm{mg}^{-1}=0.51 \mathrm{mg}$. This allows us to calculate the nanotube content of the carbon soot as $34 \%$. This value is consistent with estimates of nanotube content in various carbon soots from scanning electron microscopy and transmission electron microscopy.

In conclusion we have demonstrated a preparation method which allows us to isolate nanotubes from unwanted carbon components with a high nanotube yield. This allows us to use EPR and TGA to calculate the nanotube content (purity) of the soot. For the carbon soot used in this study, the nanotube content was $34 \%$ by mass.

While calculation of carbon soot purity is the most obvious benefit of this technique many other potential advantages exist. To date no technique exists which can give quantitative measurements of nanotube content in any environment. In the past nanotube researchers in all fields have relied on purely qualitative methods such as measuring ratios of ill-defined Raman peaks or counting nanotubes in SEM or TEM micrographs. This present technique will eradicate these issues and finally solve the fundamental problem of high yield nanotube extraction. Furthermore we believe that this work is the first step toward the ability to design specific polymer architectures to select tubes of given chirality or diameter. This is considered to be one of the most important potential developments needed to make nanoelectronics using nanotubes a reality.

The authors wish to thank the Irish Higher Educational Authority for partly funding this work.

\section{Notes and references}

1 S. J. Tans, A. R. M. Verschueren and C. Dekker, Nature, 1998, 393, 49.

2 J. Hu, M. Ouyang, P. Yang and C. M. Lieber, Nature, 1999, 399, 48.

3 J. Li, A. M. Cassell and H. Dai, Surf. Interface Anal., 1999, 28, 8.

4 P. Wagner, S. Nock, J. A. Spudich, W. D. Volkmuth, S. Chu, R. L. Cicero, C. P. Wade, M. R. Linford and C. E. D. Chidsey, J. Struct. Biol., 1997, 119, 18.

5 F. Balavoine, C. Richard, T. W. Ebbesen, C. Mioskowski and P. Schulz, Biol. Cell, 1998, 90, 283.

6 S. Bandow, J. Appl. Phys., 1996, 80, 1020.

7 T. W. Ebbesen, P. M. Ajayan, H. Hiura and K. Tanigaki, Nature, 1994, 367, 519.

8 G. S. Duesberg, M. Burghard, J. Muster, G. Philipp and S. Roth, Chem. Commun., 1998, 3, 435.

9 W. Holzer, A. Penzkofe, S. H. Gong, A. Bleyer and D. D. C. Bradley, Adv. Mater., 1996, 8, 974.

10 W. Krätschmer, L. D. Lamb, K. Fostiropoulos and D. R. Huffman, Nature, 1990, 347, 354.

11 O. Chauvet, L. Forro, W. Bacsa, D. Ugarte, B. Doudin and W. A. de Heer, Phys. Rev. B., 1995, 52, R6963. 